\title{
Bis zum Tod stabil gelagert
}

\section{Die stabile Seitenlage kann es erschweren, einen Atemstillstand rasch zu erkennen und mit einer Herzdruckmassage zu beginnen, wie spanische Forscher berichten.}

\) Zu den wichtigsten Maßnahmen in der Notfallversorgung bewusstloser Patienten gehört es, das Versagen der Atmung zu erkennen und in diesem Fall sofort mit der Herzdruckmassage zu beginnen. Hierfür wird in den Leitlinien zur Reanimation des European Resuscitation Council empfohlen, den Patienten in die HTCL (Head Tilt Chin Lift)-Position zu bringen: „Legen Sie Ihre Hand auf seine Stirn und ziehen Sie seinen Kopf leicht nach hinten; Heben Sie mit Ihren Fingerspitzen das Kinn des Patienten an, um die Atemwege frei zu machen. Drehen Sie den Patienten auf den Rücken."

Und bei bewusstlosen, spontan atmenden Patienten gilt die stabile Seitenlagerung als optimal, um eine As- piration $\mathrm{zu}$ verhindern. Was aber, wenn die zunächst spontane Atmung sistiert? Lässt sich ein sich anbahnender Atemstillstand hier gegebenenfalls rasch identifizieren?

„Nein“, meint eine Forschergruppe um den Notfallspezialisten Miguel Freire-Tellado, Lugo/Spanien. Die Wissenschaftler hatten in einer Studie 59 Teilnehmer, die in Herz-Lungen-Wiederbelebung ausgebildet waren, in zwei Gruppen eingeteilt: Eine hatte gelernt, die Patienten in stabile Seitenlage zu bringen, die zweite in die HTCLLagerung. Freire-Tellado und Kollegen stoppten, wie schnell die Probanden in einer simulierten Notfallsituation den einsetzenden Atemstillstand eines Bewusstlosen bemerkten. Binnen zwei
Minuten schafften das 14 von 27 Studienteilnehmern bei Seitenlagerung und 23 von 28 bei Lagerung in HTCLPosition.

\section{Schlechtere Einschätzung der Atemtätigkeit}

„Die stabile Seitenlage behinderte die Einschätzung der Atemtätigkeit und verzögerte das Erkennen des Atemstillstands", fassen Freire-Tellado und Mitarbeiter die Resultate ihrer Studie zusammen. Im Vergleich zur HTCL-Position habe dies den Beginn der Herzdruckmassage verzögert und die Wahrscheinlichkeit erhöht, nicht mit der kardiopulmonalen Reanimation zu beginnen.

(rb)

Quelle

Freire-Tellado $M$ et al. Resuscitation 2017, online 9. April; https://doi.org/10.1016/j. resuscitation.2017.03.008

\section{Salmonellen-Infektionen in der EU nehmen zu}

\section{Die Europäische Seuchenbehörde ist besorgt: Die Zahl der Salmo- nellosen in der EU steigt wieder.}

\) Die Europäische Seuchenbehörde ECDC spricht von einer „Mahnung, wachsam zu bleiben“: Die Zahl der Infektionen mit Salmonella (S.) enteriditis nimmt wieder $\mathrm{zu}$ - nachdem die Fallzahlen seit 2008 rückläufig waren. Im Vergleich zu 2014 gab es 2016 3\% mehr Fälle, wie die Behörde im Zoonosen-Bericht 2016 (doi: 10.2903/j.efsa.2017.5077) informiert. 0,25\% der Infektionen verliefen tödlich.

„Der Rückgang der Salmonellen-Infektionen war eine Erfolgsgeschichte für die Überwachung der Lebensmittelsicherheit innerhalb der EU“, wird Marta Hugas, Europäische Behörde für Lebensmittelsicherheit EFSA, die den Bericht gemeinsam mit der ECDC herausgegeben hat, zitiert. Zur Umkehr von diesem Trend hätten mehrere Salmonellen-Ausbrüche in den letzten Jahren beigetragen. Das ECDC berich- tet, von den 4.786 nachweisbar durch den Verzehr kontaminierter Nahrung verursachten Zoonosen in der EU sei jede sechste auf den Erreger S. enteriditis zurückzuführen. Risikofaktoren sind besonders der Verzehr von Eiern und Eiprodukten. „Um die Gründe für die Zunahme allerdings richtig zu erfassen, sind weitere Untersuchungen der jeweiligen Behörden für Lebensmittelsicherheit nötig“, so Hugas.

Insgesamt wurden 2016 in der EU 94.530 Salmonellen-Infektionen gemeldet - damit lag die Salmonellose auf Platz zwei der häufigsten Zoonosen. Häufiger waren nur Campylobacter-Infektionen, von denen 246.307 gemeldet wurden -im Vergleich zu 2015 eine Zunahme von 6\%. Der Durchfall-Erreger selbst ist dabei eher harmlos: 0,03\% aller Infektionen verliefen tödlich. Allerdings ist die Zahl der Hospitalisationen nicht unbedingt gering: Immerhin 29\% der Patienten mussten in ein Krankenhaus eingeliefert werden.

Risikofaktor für eine Infektion ist dabei vor allem der Verzehr von kontaminiertem Fleisch, erinnert das ECDC. Der größte Campylobacter-Ausbruch sei 2016 aus Schweden gemeldet worden. Ursache: der Verzehr von verunreinigtem Geflügelfleisch.

Deutlich gefährlich sind Infektionen mit Listerien: Von den $2.536 \mathrm{im} \mathrm{Jahr}$ 2016 in der EU gemeldeten Fälle verliefen $16,2 \%$ tödlich - damit ist die Listeriose die gefährlichste der im Bericht erfassten Zoonosen. Zudem musste beinahe jeder Patient (98\%) in eine Klinik gebracht werden. Insgesamt starben 247 EU-Bürger im Jahr 2016 an einer Listeriose, die meisten waren über 64 Jahre alt. Bei den Patienten über 84 Jahren starb mehr als jeder Vierte.

(Anne Bäurle)

Quelle:

www.springermedizin.de 